\title{
The Development and Verification for Reliability and Validity of a Japanese Version of Greene et al.'s Moral Dilemma Questionnaire
}

\section{YOSHIYUKI TAKIMOTO ( $\sim$ takimoto@m.u-tokyo.ac.jp )}

The University of Tokyo Graduate School of Medicine Faculty of Medicine: Tokyo Daigaku Daigakuin Igakukei Kenkyuka Igakubu https://orcid.org/0000-0001-7033-2623

\section{Akira Yasumura}

Kumamoto University: Kumamoto Daigaku

\section{Short report}

Keywords: moral psychology, moral dilemma, questionnaire development

Posted Date: March 1st, 2022

DOI: https://doi.org/10.21203/rs.3.rs-1393128/v1

License: (c) (i) This work is licensed under a Creative Commons Attribution 4.0 International License.

Read Full License 


\section{Abstract}

Background: A difference exists in people's judgements between the switch case and pedestrian bridge case of the trolley problem, which is explained by the concept of personalness or directness. The moral dilemma task questionnaire developed by Greene, which comprises personal and impersonal moral dilemmas, is useful for clarifying people's moral judgements. However, the validity of Green's questionnaire was not validated. We developed and validated a Japanese version of this questionnaire.

Methods and Results: Ten new questions were added to the Japanese version using double translation and its internal validity was tested. A second survey was conducted on the same participants one month after the first survey $(n=231)$. The intraclass correlation coefficient by retest was found to be 0.781 .

Conclusion: Reproducibility, internal consistency, and criterion-related validity were confirmed by retesting the Japanese version of the moral dilemma task by Greene et al. The current study proved the reliability of the original version.

\section{Introduction}

In the last two decades, empirical research based on the moral emotionalism theory has advanced the psychological elucidation of our moral judgments [1]. In this context, the moral dilemma issue, represented by the trolley problem [2,3], has played a pivotal role. It is a moral predicament similar to the ethical dilemma of whether to follow a consequentialist or an intuitivist judgment.

In the trolley problem, people's judgments in the switch and the footbridge cases are different [4], and this difference is explained by the concept of "personalness" or "directness" [5, 6]. The concept of "personalness" or "directness" in moral dilemmas around the use of violence is one that involves (a) serious physical harm (b) to one or more specific individuals, (c) when it is not the result of averting an existing threat [5]. In the trolley problem, the switch case is an impersonal moral dilemma, and the act of pressing the switch would be unlikely to be accompanied by any emotion. Therefore, people would choose to sacrifice one person to save five without any psychological struggle. Contrarily, the footbridge case is a personal moral dilemma, and the act of pushing a person off the footbridge would be accompanied by emotion. Therefore, people are unlikely to opt for sacrificing one fat person to save five railroad workers because of psychological resistance. The concept of "personalness" or "directness" forms a core part of the moral emotionalism theory.

Greene et al. used functional Magnetic Resonance Imaging (fMRI) in their first study to measure brain activity during a moral dilemma task and explained the difference in judgment between the personal and impersonal moral dilemmas in terms of different emotional responses [7]. Later, the dual-process theory was applied to explain it as a difference in the neural system of involuntary (autonomic) and voluntary (somatic) reactions, whereby the moral dilemma is based on the competition between these reactions [8]. 
The relationship between "personalness" or "directness" of emotional responses and the moral judgments based on them, reflects human biological characteristics and is cross-cultural. Cushman et al. [4] conducted a large web-based survey with more than 5,000 respondents and found insignificant effects of education level, religious background, and ethnicity on completing the dilemma task. The universality of "personalness" or "directness" in moral judgments can indicate the variety of moral judgments that arise due to developmental and organic variations in the brain. For example, in a comparative study involving three groups (frontotemporal dementia subjects, Alzheimer's Disease dementia subjects, and healthy volunteers), no difference between them was noted in the switch case, with at least $80 \%$ of the subjects in each of the three groups indicating that they would throw the switch and save five people. However, the footbridge case was favored by approximately $20 \%$ of the participants with Alzheimer's dementia and healthy volunteers and nearly $60 \%$ of the participants with frontotemporal dementia [8].

The moral dilemma task used in Greene et al.'s study consisted of 64 dilemma questions categorized as personal moral, impersonal moral, and non-moral, including trolley problems in the switch and footbridge cases. In order to use "personalness" or "directness" as indicators of moral judgment variation, it is necessary to verify their reliability and validity as an indicator. Although Greene et al.'s moral dilemma task is useful as a measure of "personalness" or "directness," it has not been tested for reliability or validity. Therefore, the purpose of this study was to create a Japanese version of the moral dilemma task and examine it from this point of view. Furthermore, we aimed to investigate the differences in moral judgments among Japanese speakers and to add knowledge about linguistic universality to the findings of Cushman et al. [4]. Proving the reliability and validity of the Japanese version will be relevant for the English version as well. As a result, Greene et al.'s moral dilemma questionnaire is expected to contribute to the establishment of an index for measuring differences in moral judgments associated with developmental and organic brain variations.

\section{Method}

\subsection{Overview of the Moral Dilemma Question}

The moral dilemma questionnaire [7] consists of 64 questions divided into three categories. The first category comprises : 1) personal moral dilemmas (e.g., the footbridge problem), which involve physical contact or other direct sacrifice of the few to help the many; 2 ) impersonal moral dilemmas (e.g., the trolley problem), which involve indirect sacrifice of the few to help the many; and 3) impersonal moral dilemmas (e.g., the trolley problem), which involve indirect sacrifice of the many to help the few. Altogether, these three subcategories comprise 25 questions.

The second category includes impersonal moral dilemmas (e.g., the trolley problem), which involve indirectly helping the many at the expense of the few (19 questions), and the third category comprises non-moral issues (20 questions). The response format is a four-point scale: $1=$ not appropriate, $2=$ somewhat inappropriate, 3 = somewhat appropriate, and 4 = appropriate. In addition, there were 19 reversed items. 


\subsection{Procedure for Creating the Japanese Version of the Moral Dilemma Problem}

To create the Japanese version of the moral dilemma questionnaire, the authors obtained the approval of the original version's developers. First, the authors translated the questions into Japanese, and then the questions were back-translated by a native speaker who did not know the original text. While comparing the back-translation with the original text, the authors, who are experts in ethics, revised the Japanese version. Furthermore, a back-translation was done by a native speaker who did not know the original text to confirm the corrected Japanese.

The results of the second back-translation were presented to the developers, who confirmed a general good level of accuracy. They made the necessary corrections in the form of comments on items with problematic expressions, and provided a final approval when all issues were solved.

\subsection{Modification and Addition of Items}

Three questions were deleted, as instructed by the developers. One question was deleted because of cultural differences between schools in the United States of America and Japan. As a result, there were 22 personal moral dilemmas, 19 questions related to impersonal moral dilemmas and 19 unrelated to morality. Furthermore, ten of the impersonal moral dilemma tasks (Q30-39) and ten of the non-moralrelated tasks (Q11-20) were set as reversed items. For the purpose of validation, four personal, and four impersonal moral dilemma questions (Q65-72), which were created under the trolley problem, were added as additional items only in the second application of the survey, a month after the first.

\subsection{Survey Participants and Methods}

A total of 364 people, who had read the explanation of, and agreed to participate in the study, registered with the research company (Macromill, Inc). The participants included 120 men and 111 women. The mean age was 40.2 years (SD \pm 11.3 , range 20.0 to 59.0) (Table 1). The selection criteria were Japanese nationality and lack of disabilities, such as mental illness. To eliminate regional differences, the participants were recruited equally from all regions of Japan. A web-based survey was conducted by Macromill, Inc. To verify reliability, a second survey was conducted with the same participants one month after the first. The identity of the participants was anonymized.

\section{Table 1}

Participant Characteristics 


\begin{tabular}{ll} 
Gender (N) & \\
\hline Male & 120 \\
\hline Female & 231 \\
\hline Total & \\
\hline Regions (N) & 29 \\
\hline Hokkaido & 25 \\
\hline Tohoku & 35 \\
\hline Kanto & 34 \\
\hline Chubu & 25 \\
\hline Kinki & 31 \\
\hline Chugoku & 27 \\
\hline Shikoku & 25 \\
\hline Kyushu[Okinawa & 231 \\
\hline Total & \\
\hline Age (years) & 40.2 \\
\hline Mean & 11.3 \\
\hline SD & $20-59$ \\
\hline Range & \\
\hline S of he wo & \\
\hline
\end{tabular}

Participants who completed only one out of the two surveys were excluded from the analysis.

Respondents with a small variation in answers to all questions (standard deviation of 0.2 or less), and respondents whose time required to complete the survey was less than $1 / 16$ th of the total time, were excluded from the analysis because they were likely to have answered the questions inadequately. Using the above exclusion criteria, a total of 231 remaining cases were considered valid responses.

\subsection{Analysis Method}

To verify reliability, Cronbach's alpha coefficient was obtained as an index of internal consistency, and intraclass correlation coefficients (ICC) were calculated as a measure of reproducibility. Additionally, the criterion-related validity calculated from the means of Greene's moral dilemma tasks and impersonal moral dilemma tasks and the means of additional personal moral dilemma tasks and impersonal moral dilemma tasks of the trolley problem were determined. Furthermore, to examine differences by region, an analysis of variance was conducted across regions by the first category. 


\section{Results}

\subsection{Reliability and Validity}

The reliability of the survey was verified using a test-retest method. Cronbach's alpha coefficient for all items in the first application was 0.821 and in the second application was 0.885 (excluding the additional items) (Fig. 1).

The ICC $(1,2)$ for all the items was 0.795 (95\% Cl: 0.735, 0.842) (Table 2).

Table 2

Internal consistency and Reliability by Retest Method

\begin{tabular}{llll} 
& Study 1 & Study 2 & \\
\hline & Cronbach's a & Cronbach's a & ICC $(1,2)(95 \% \mathrm{Cl})$ \\
\hline NM (Q1-20; Q14 excluded) & 0.791 & 0.824 & $0.794(0.733,0.841)$ \\
\hline MI (Q21-39) & 0.624 & 0.677 & $0.722(0.640,0.785)$ \\
\hline MP (Q40-64; Q44, 47, 53 excluded) & 0.876 & 0.910 & $0.850(0.806,0.884)$ \\
\hline ALL (Q1-64; Q14 \& Q44, 47, 53 excluded) & 0.823 & 0.864 & $0.795(0.735,0.842)$
\end{tabular}

Note. NM: non-moral issue; MI: impersonal moral dilemma task; MP: personal moral dilemma task

The criterion validity testing revealed a strong positive correlation between the first and additional items in the personal moral dilemma questions $(r=0.653, p<.00001)$. A weak negative correlation was found for the impersonal moral dilemma questions $(r=-0.226, p<.0001)$.

\subsection{Differences by Region}

There were no regional differences in all items or in any category of questions (all items: $F(7,223)=$ 1.337, $p=0.23$; personal moral dilemma tasks: $F(7,223)=0.433, p=0.881$; impersonal moral dilemma tasks: $F(7,223)=0.699, p=0.673$; non-moral related tasks: $F(7,223)=1.110, p=0.361)$.

\section{Discussion}

The purpose of this study was to create a Japanese version of Greene et al.'s moral dilemma questionnaire [7], through the process of translation and back-translation.

A test-retest method was used to verify reliability. The ICC for all items was 0.781 , and Cronbach's alpha coefficient was 0.821 for the first test and 0.885 for the second test, suggesting that the Japanese version 
of the moral dilemma task was highly reliable.

Regarding content validity, considering that the purpose of the study was to create a Japanese version, we used expressions that are more familiar to Japanese speakers (for example, "meters" instead of "yards," "rice cutter," etc.)

For criterion-related validity, the case of the railroad bridge in the trolley problem was used as an indicator of the personal moral dilemma task, and the case of the switcher in the trolley problem was used as an indicator of the impersonal moral dilemma task. A certain degree of validity was obtained for each participant. However, the bridge case and the switch case can be interpreted not only as differences in terms of "personalness" but also as differences in whether people are used as a means, and whether or not the action is intentional [9]. From this point of view, whether these two cases are valid external indicators of criterion-related validity requires further discussion.

Regarding regional differences, none were observed in Japan, suggesting that uniform results can be obtained in any region of the country. This result was consistent with that of Hauser et al.'s study [10].

\section{Conclusion}

Using a Japanese version of Greene et al.'s moral dilemma questionnaire with sufficient reliability and validity, the current study proved the reliability of the original version. Furthermore, no regional differences were found within Japan, suggesting the possibility of obtaining common results across diverse cultures.

\section{Declarations}

Acknowledgements. The authors thank Eisuke Nakazawa for cooperation in data collection, advice on experimental design, and Editage corp. for editing the manuscript and funding support by AMED.

Funding: This research was supported by (AMED) under Grant Number SRPBS 16769942.

Declaration of Conflicting Interests: The authors declare that there is no conflict of interest associated with this manuscript.

Compliance with Ethical Standards: All procedures followed were in accordance with the ethical standards of the responsible committee on human experimentation (institutional and national) and with the Helsinki Declaration of 1975 (in its most recently amended version). This study was approved by the Ethics Committee of the Faculty of Medicine, The University of Tokyo (No. 1468-1).

Informed Consent. Informed consent was obtained from all participants in the study.

Availavility pf data and materilals: The datasets used and/or analysed during the current study are available from the corresponding author on reasonable request. 
Author's Contribution: Yoshiyuki Takimoto and Akira Yasumura contributed to the design and implementation of the research, to the analysis of the results and to the writing of the manuscript.

\section{References}

1. Prinz J. The emotional construction of morals. Oxford: Oxford University Press; 2007.

2. Foot P. The problem of abortion and negative and positive duty: a reply to James LeRoy Smith. J Med Philos. 1978;3:253-5. https://doi.org/10.1093/jmp/3.3.253.

3. Thomson JJ. The trolley problem [Unpublished manuscript]. Yale Law J. 1985;94. https://doi.org/10.2307/796133.

4. Cushman $F$, Young $L$, Hauser $M$. The role of conscious reasoning and intuition in moral judgment: testing three principles of harm. Psychol Sci. 2006;17:1082-9. https://doi.org/10.1111/j.14679280.2006.01834.x.

5. Greene JD, Morelli SA, Lowenberg K, Nystrom LE, Cohen JD. Cognitive load selectively interferes with utilitarian moral judgment. Cognition. 2008;107:1144-54. https://doi.org/10.1016/j.cognition.2007.11.004.

6. Greene JD, Cushman FA, Stewart LE, Lowenberg K, Nystrom LE, Cohen JD. Pushing moral buttons: the interaction between personal force and intention in moral judgment. Cognition. 2009;111:36471. https://doi.org/10.1016/j.cognition.2009.02.001.

7. Greene JD, Sommerville RB, Nystrom LE, Darley JM, Cohen JD. An fMRI investigation of emotional engagement in moral judgment. Science. 2001;293:2105-8. https://doi.org/10.1126/science.1062872.

8. Kahneman D. A perspective on judgment and choice: mapping bounded rationality. Am Psychol. 2003;58:697-720. https://doi.org/10.1037/0003-066X.58.9.697.

9. Young L, Tranel D, Cushman F, Hauser M, Adolphs R. Does emotion mediate the relationship between an action's moral status and its intentional status? Neuropsychological evidence. J Cogn Cult. 2006;6:291-304. https://doi.org/10.1163/156853706776931312.

10. Hauser MD, Cushman FA, Young LL, Kang-xing Jin R, Mikhail JA. A dissociation between moral judgments and justifications. Mind Lang. 2007;22:1-21. https://doi.org/10.1111/j.14680017.2006.00297.x.

\section{Further reading.}

1. Mendez MF, Anderson E, Shapira JS. An investigation of moral judgement in frontotemporal dementia. Cogn Behav Neurol. 2005;18:193-7. https://doi.org/10.1097/01.wnn.0000191292.17964.bb.

\section{Figures}




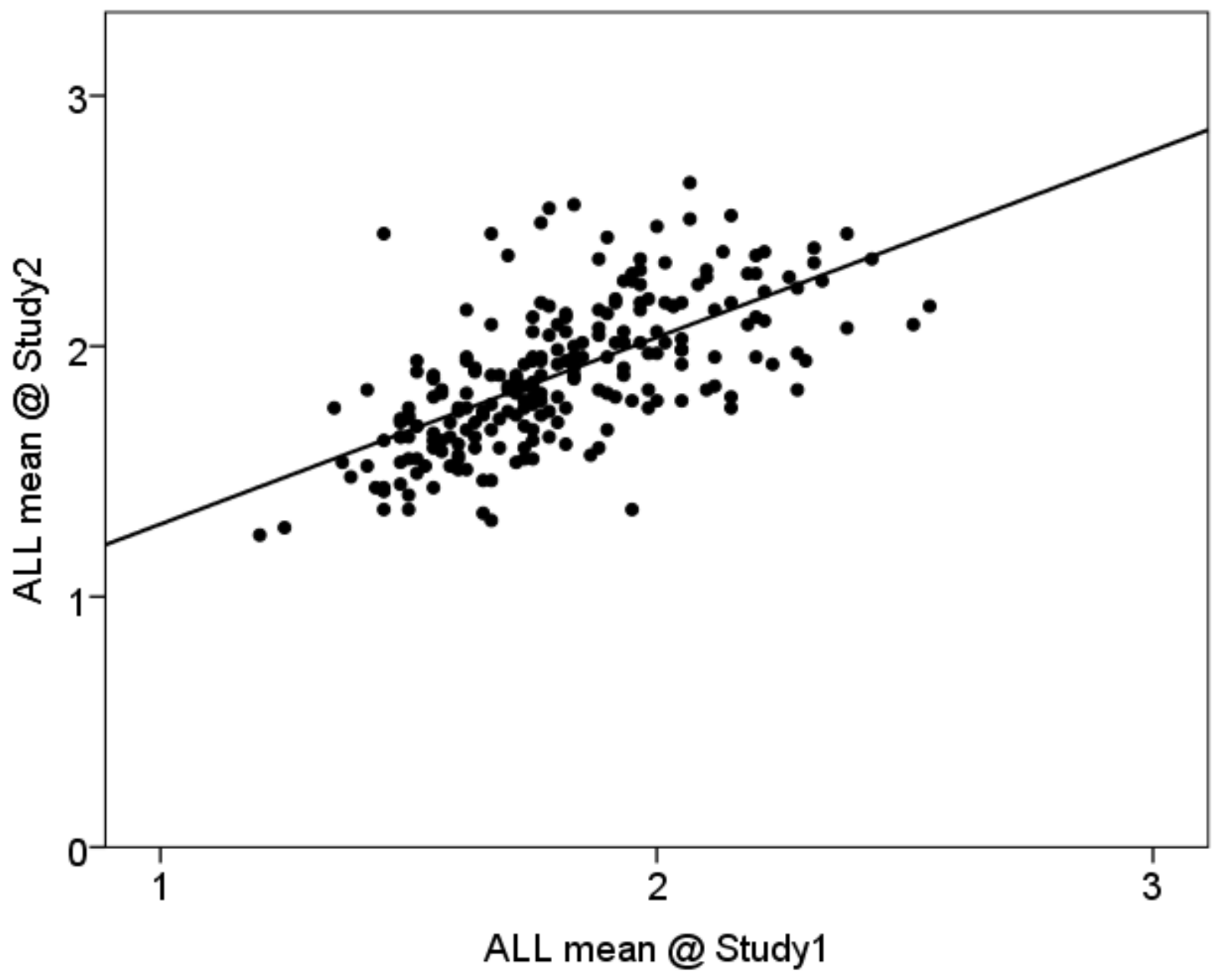

Figure 1

The Correlation of Mean between Study 1 and Study 2 